\title{
Criminologie
}

\section{Itinérance, santé mentale, justice} Expérience et perceptions des utilisateurs de services à Montréal

\section{Homelessness, mental health and justice}

Experiences and perceptions of service users in Montréal

Indigencia, salud mental, justicia

Experiencia y percepciones de los usuarios de servicios en Montreal

\author{
Laurence Roy, Marichelle C. Leclair, Michelle Côté et Anne G. Crocker
}

Volume 53, numéro 2, automne 2020

URI : https://id.erudit.org/iderudit/1074199ar

DOI : https://doi.org/10.7202/1074199ar

Aller au sommaire du numéro

Éditeur(s)

Les Presses de l’Université de Montréal

ISSN

0316-0041 (imprimé)

1492-1367 (numérique)

Découvrir la revue

Citer cet article

Roy, L., Leclair, M. C., Côté, M. \& Crocker, A. G. (2020). Itinérance, santé mentale, justice : expérience et perceptions des utilisateurs de services à Montréal. Criminologie, 53(2), 359-383. https://doi.org/10.7202/1074199ar

\section{Résumé de l'article}

Plusieurs programmes et services ont été développés en vue d'adapter les interventions policières et les processus judiciaires aux besoins des personnes ayant des troubles mentaux, de surcroît lorsque celles-ci sont en situation d'itinérance. La présente étude adopte un devis qualitatif descriptif afin d'explorer l'expérience qu'ont les personnes vivant à la fois une situation d'itinérance et un trouble mental de ces services. L'analyse de six entretiens révèle les représentations complexes que se font les participants de leur implication judiciaire, entre sentiment de responsabilité et d'injustice ; le manque de légitimité vécu dans la plupart de leurs interactions, auquel l'accompagnement offre parfois un contrepoids ; et enfin des perceptions distinctes des services selon leur nature " régulière » ou " alternative ». Les participants mettent à l'avant-plan dans leurs récits les principes de la justice procédurale, en particulier ce que des processus dits « alternatifs " permettent à cet égard, mais également le caractère exceptionnel d'interactions respectant ces principes. Les résultats nous amènent à interroger la capacité des diverses institutions sociales à offrir des services vécus comme justes par les personnes situées au confluent d'identités sociales marginalisées, à différents moments de leurs parcours. 


\title{
Itinérance, santé mentale, justice Expérience et perceptions des utilisateurs de services à Montréal
}

\author{
Laurence Roy ${ }^{1,2}$ \\ Professeure agrégée, École de physiothérapie et d'ergothérapie \\ Université McGill \\ Institut universitaire en santé mentale Douglas \\ laurence.roy@mcgill.ca

\section{Marichelle C. Leclair} \\ Doctorante, Département de psychologie \\ Université de Montréal \\ Institut national de psychiatrie légale Philippe-Pinel \\ marichelle.leclair@umontreal.ca

\section{Michelle Côté} \\ Chercheuse régulière \\ Institut national de psychiatrie légale Philippe-Pinel \\ michellecote@ville.montreal.qc.ca
}

\section{Anne G. Crocker}

Professeure titulaire, Département de psychiatrie et d'addictologie Université de Montréal

Institut national de psychiatrie légale Philippe-Pinel

anne.crocker@umontreal.ca

RÉSUMÉ - Plusieurs programmes et services ont été développés en vue d'adapter les interventions policières et les processus judiciaires aux besoins des personnes ayant des troubles mentaux, de surcroît lorsque celles-ci sont en situation d'itinérance. La présente étude adopte un devis qualitatif descriptif afin d'explorer l'expérience qu'ont

1. École de physiothérapie et d'ergothérapie, Université McGill, 3654, Promenade Sir-William-Osler, Montréal (Québec), Canada, H3G 1 Y5.

2. Laurence Roy a obtenu le soutien financier du Fonds de recherche du Québec - Santé (FRQ-S) sous forme de bourse Chercheur-boursier Junior 1 pendant la durée de ce projet. Marichelle Leclair a obtenu le soutien financier du FRQ-S puis celui des Instituts de recherche en santé du Canada sous forme de bourse doctorale Vanier et celui de l'Observatoire en justice et santé mentale pendant la durée de ce projet. Ce projet a été financé par une subvention des Instituts de recherche en santé du Canada.

Criminologie, vol. 53, $\mathrm{n}^{\circ} 2$ (2020) 
les personnes vivant à la fois une situation d'itinérance et un trouble mental de ces services. L'analyse de six entretiens révèle les représentations complexes que se font les participants de leur implication judiciaire, entre sentiment de responsabilité et d'injustice; le manque de légitimité vécu dans la plupart de leurs interactions, auquel l'accompagnement offre parfois un contrepoids; et enfin des perceptions distinctes des services selon leur nature «régulière» ou "alternative». Les participants mettent à l'avant-plan dans leurs récits les principes de la justice procédurale, en particulier ce que des processus dits "alternatifs» permettent à cet égard, mais également le caractère exceptionnel d'interactions respectant ces principes. Les résultats nous amènent à interroger la capacité des diverses institutions sociales à offrir des services vécus comme justes par les personnes situées au confluent d'identités sociales marginalisées, à différents moments de leurs parcours.

MotS CLÉs - Itinérance, judiciarisation, police, troubles mentaux.

\section{Introduction}

Les personnes vivant avec un trouble mental sont plus susceptibles que les personnes sans trouble mental d'être en contact avec les services policiers (Charette, Crocker et Billette, 2011; Hartford, Heslop, Stitt et Hoch, 2005 ; Livingston, 2016), d'être impliquées avec la justice pénale (Lurigio, 2012; Markowitz, 2011), et d'être incarcérées (Lynch et al., 2014; Prins, 2014). De surcroît, la situation d'itinérance ou d'instabilité résidentielle amplifie les probabilités d'interactions policières et judiciaires (Roy et al., 2016; Roy, Crocker, Nicholls, Latimer et Ayllon, 2014). Une recension systématique des écrits révèle que la majorité des personnes en situation d'itinérance ayant un trouble mental ont été mises en état d'arrestation au cours de leur vie, et que plus de la moitié ont été incarcérées (Roy et al., 2014).

L'implication judiciaire, cumulée au trouble mental et à la situation d'itinérance, a des conséquences importantes sur les trajectoires de vie des personnes. On sait, par exemple, que les personnes ayant des troubles mentaux sont plus susceptibles d'être victimisées lors d'une incarcération (Choe, Teplin et Abram, 2008), ce qui peut provoquer ou exacerber des problèmes de santé mentale et physique. L'implication judiciaire entraînerait également une fragmentation ou un arrêt des suivis médicaux, psychiatriques et psychosociaux (Copeland et al., 2009), ainsi qu'un phénomène de stigmatisation cumulée liée non seulement au trouble mental et au statut de pauvre ou d'itinérant, mais également aux étiquettes de «criminel» ou «délinquant» (Batastini, Bolanos et Morgan, 2014; Frappier, Vigneault et Paquet, 2009). Ensemble, ces facteurs tendent à prolonger les épisodes d'itinérance des 
personnes ayant des troubles mentaux lorsque l'implication judiciaire survient (Caton et al., 2005; Levitt, Culhane, DeGenova, O'Quinn et Bainbridge, 2009), et diminuent les perspectives d'intégration sociale lors d'une stabilisation éventuelle de la situation résidentielle (Frounfelker, Glover, Teachout, Wilkniss et Whitley, 2010; Poremski, WoodhallMelnik, Lemieux et Stergiopoulos, 2015).

Bien que l'ampleur du phénomène de judiciarisation des personnes cumulant situation d'itinérance et trouble mental, ainsi que ses conséquences, fasse l'objet d'un relatif consensus scientifique, les angles d'analyse diffèrent. À un pôle, plusieurs études adoptent une approche principalement clinique afin d'expliquer la judiciarisation en mettant de l'avant des facteurs individuels et interpersonnels comme l'impulsivité, la toxicomanie et les traumatismes émotionnels (Crawford, Whitbeck et Hoyt, 2011 ; Edalati et al., 2020; Leclair et al., 2020; Roy et al., 2016). D'autres travaux mettent l'accent sur le rôle des appareils policier et judiciaire, par leur fonction de contrôle social de populations «hors norme», dans le maintien des taux d'implication judiciaire élevés (Chesnay, Bellot et Sylvestre, 2013 ; Sylvestre, 2010; Sylvestre, Bernier et Bellot, 2015). Dans les dernières décennies, plusieurs pratiques décentralisées de régulation des populations en situation d'itinérance ont été ainsi mises en place, par exemple la multiplication des règlements municipaux dans le cadre de la «lutte aux incivilités», ce qui a laissé libre cours aux pratiques informelles de profilage social, et les modifications urbaines et architecturales visant à rendre invisibles ou repousser ces populations (Commission des droits de la personne et des droits de la jeunesse du Québec, 2009; Nault, Couture-Glassco et Larose-Hébert, 2016; Sylvestre, Bellot, Ménard et Tremblay, 2011; Sylvestre, Blomley et Bellot, 2020). Autant dans l'intervention et les politiques publiques que dans les travaux scientifiques, des tensions émergent entre une représentation de l'itinérance où l'étiquette du trouble mental est mobilisée, et une représentation qui préconise plutôt celle de la déviance (Roy, Lemieux et Crocker, 2019; Sylvestre, Bellot et Chesnay, 2012). À cette tension s'ajoute celle des réponses à préconiser: dans le premier cas, une approche axée sur le rétablissement, favorisant l'autodétermination et l'accompagnement des personnes; dans le deuxième, la surveillance accrue et le contrôle de ces populations.

Au Québec comme ailleurs dans le monde, divers programmes ont été développés en vue d'adapter les interventions policières et les processus judiciaires aux besoins des personnes ayant des troubles mentaux 
et, dans certains cas, à diriger ces personnes vers des suivis (traitement, accompagnement psychosocial) jugés plus adéquats que l'incarcération. Ces programmes, principalement d'inspiration américaine, incluent des équipes mixtes policiers-intervenants qui agissent ensemble auprès de personnes ayant des troubles mentaux (Kisely et al., 2010), la formation des policiers à la réponse en intervention de crise (Wood et Watson, 2016) et des programmes de solutions de rechange à l'incarcération de type tribunaux spécialisés (Schneider, Crocker et Leclair, 2016).

\section{Le contexte montréalais}

À Montréal, la mise en œuvre de tels programmes s'est effectuée dans un contexte particulier. Entre 2009 et 2015, des patrouilleurs montréalais ont été impliqués dans plusieurs interactions s'étant soldées par la mort de personnes en situation d'itinérance ayant des troubles mentaux (Coroner du Québec, 2012, 2016). Ces évènements, en parallèle à une prise de conscience de la fréquence des contacts entre policiers et personnes ayant des troubles mentaux (Charette et al., 2011), ont mené, en quelques années, à l'émergence d'une panoplie de nouveaux programmes offrant des options alternatives à l'arrestation ou à l'incarcération. Deux équipes mixtes policiers-intervenants ont été mises en place. L'Équipe de soutien aux urgences psychosociales (ÉSUP) intervient auprès de personnes dont l'état mental semble perturbé, sans égard à leur statut résidentiel. L'Équipe mobile de référence et d'intervention en itinérance (ÉMRII) assure l'intervention et le suivi auprès des personnes en situation d'itinérance générant de nombreux appels policiers. Une centaine de patrouilleurs montréalais ont été formés à l'approche «Réponse en intervention de crise» (RIC) depuis 2013 afin d'améliorer la détection des troubles mentaux par les policiers, le recours aux techniques de pacification et l'arrimage avec les services sociosanitaires. Ces innovations s'ajoutent à des services déjà existants, dont le programme Urgence psychosocialejustice soutenant, depuis 1996, les intervenants de proximité, entre autres, auprès de personnes en crise. Divers programmes d'accompagnement ont également été établis à la cour municipale de Montréal (Fortin et Raffestin, 2017; Provost, 2011). Enfin, des organismes communautaires montréalais jouent un rôle d'accompagnement et de défense des droits des personnes marginalisées auprès des instances judiciaires (St-Jacques, 2016). 


\section{Cadre théorique}

Dans la majorité des cas, les programmes développés à Montréal et ailleurs se sont réclamés de la théorie de la justice procédurale (Tyler, 1990, 2009; Tyler et Fagan, 2008). Celle-ci distingue la perspective instrumentale de la perspective normative dans le rapport à l'autorité et aux règles. Selon Tyler, la perspective instrumentale reste centrée sur les gains personnels et les conséquences négatives ou positives de se conformer ou non aux règles dans une situation donnée. Dans la perspective normative, la décision de se conformer aux règles trouve plutôt sa source principalement dans la légitimité perçue des règles (en fonction des valeurs et normes personnelles) et des figures d'autorité chargées de les appliquer et de les sanctionner. Les personnes percevraient donc leurs interactions avec des figures d'autorité comme plus ou moins «justes» en fonction de la façon dont les règles sont mobilisées et dont les décisions sont prises, au-delà de la résultante de l'interaction. Tyler argue qu'un sentiment accru de justice procédurale favorise la coopération avec les figures d'autorité dans une situation donnée, mais également, à long terme, le respect des règles (par exemple, des conditions de libération) et la cohésion au sein d'un groupe social. De nombreux travaux empiriques réalisés auprès de différents groupes ont permis de dégager certaines dimensions clés de la justice procédurale: 1) la personne en situation d'autorité se place dans une posture d'écoute, de respect et d'accueil; 2) elle tient compte de la complexité de la situation et des responsabilités partagées par la personne elle-même, mais aussi par les intervenants sociosanitaires et acteurs judiciaires; 3) elle fait preuve de transparence en expliquant ses actions et les raisons sousjacentes à ses décisions; 4) elle favorise la participation active des personnes au processus décisionnel (Dollar, Ray, Hudson et Hood, 2018; Furness, Maguire, Brown et McKenna, 2016; Guiresse, Senon et Rossini, 2019; Livingston, Crocker, Nicholls et Seto, 2016; Mahoney, 2014; Tyler, 1990; Wales, Hiday et Ray, 2010; Wood et Watson, 2016). Dans le contexte des interactions entre personnes ayant des troubles mentaux et figures d'autorité, la justice procédurale réfère à une procédure, policière ou judiciaire, perçue comme «découlant d'un processus équitable et respectueux, auquel le principal intéressé a participé, et au cours duquel il a été respecté et traité dignement» (Guiresse et al., 2019, p. 899). Le sentiment de justice procédurale serait lié à une résolution plus positive des interactions entre personnes ayant des troubles mentaux et policiers (Wood et Watson, 2016), ainsi qu'à 
l'achèvement de programmes de solutions de rechange à l'incarcération (Dollar et al., 2018). Dans l'ensemble toutefois, ces études ont porté sur des programmes précis, qui souvent ne représentent qu'une fraction des expériences des utilisateurs de services en matière de justice. C'est d'autant plus le cas pour les personnes cumulant trouble mental et situation d'itinérance qui, à quelques exceptions près (voir Welsh et Abdel-Samad [2018], par exemple), sont peu représentées dans ce champ de recherche.

La présente étude mobilise donc le cadre théorique de la justice procédurale pour aborder l'expérience qu'ont les personnes vivant à la fois une situation d'itinérance et un trouble mental de leurs interactions policières et judiciaires, dans un contexte urbain où cohabitent des processus policiers et judiciaires «réguliers» et «alternatifs». Les questions de recherche suivantes ont été abordées: Quelle expérience des programmes ou interventions visant à éviter ou diminuer les contacts judiciaires vivent les personnes en situation d'itinérance ayant un trouble mental? Comment perçoivent-elles les actions des différents acteurs sur leur situation judiciaire, et les effets négatifs ou positifs de ces actions?

\section{Méthodes}

Un devis qualitatif descriptif (Neergaard, Olesen, Andersen et Sondergaard, 2009; Sandelowski, 2010) a été utilisé pour répondre aux questions de recherche. Cette approche qualitative vise à décrire l'expérience des participants à la recherche, de leur point de vue, en mettant de l'avant leur propre discours (Neergaard et al., 2009), et se veut donc moins interprétative que d'autres approches qualitatives. Malgré cela, cette approche n'est pas uniquement descriptive, les chercheuses offrant un éclairage théorique (Sandelowski, 2010) - ici, les concepts de justice procédurale - dans l'interprétation des données.

Pour participer, les personnes devaient avoir 18 ans ou plus, être aptes à s'exprimer en français ou en anglais et avoir un vécu d'itinérance, de trouble mental et de contacts judiciaires. Le recrutement s'est effectué en collaboration avec les responsables clinico-administratifs du réseau public de santé et de services sociaux et d'organismes communautaires, qui faisaient part de la nature du projet aux utilisateurs de leurs services correspondant aux critères recherchés. Ceux-ci pouvaient ensuite communiquer avec l'équipe de recherche pour organiser une rencontre. Un 
guide d'entretien rédigé par les membres de l'équipe de recherche abordait l'expérience qu'avaient les personnes de leurs contacts judiciaires, en particulier en ce qui a trait aux interactions avec les services policiers, judiciaires (personnel des tribunaux) et sociosanitaires. Une assistante de recherche diplômée en psychologie et en droit, et formée à la conduite d'entretiens qualitatifs, a mené tous les entretiens au moment et à l'endroit choisis par les participants. Le projet a reçu l'approbation éthique du comité d'éthique de la recherche en dépendances, inégalités sociales et santé publique du CIUSSS du Centre-Sudde-l'Île-de-Montréal. Les participants ont reçu une compensation financière de 20 \$ pour leur participation aux entretiens.

\section{Analyse des données}

Les deux premières autrices ont analysé les données selon les principes de l'analyse générale inductive (Blais et Martineau, 2006). Nous avons d'abord retranscrit intégralement dans un format commun les entretiens, puis nous nous sommes immergées dans les données, en relisant plusieurs fois les verbatim et en rédigeant des mémos sur les liaisons conceptuelles émergentes. Un résumé d'environ deux à trois pages a ensuite été créé pour chaque entrevue à partir des passages les plus saillants. À partir de ces mémos et résumés, nous avons produit une liste de codes, entendus comme des unités de sens, liés aux questions de recherche. Ensuite, de façon itérative, les codes ont été organisés et regroupés en thèmes et en sous-thèmes. Les thèmes ont ensuite été révisés, notamment en relisant les extraits liés à chaque thème et en s'assurant de leur cohérence. Les techniques de validation de contenu déployées comprennent l'utilisation de mémos théoriques par l'intervieweuse et les analystes, des séances de discussion sur les données émergentes et la validation du codage entre les deux analystes (Maxwell, 2005 ; Miles et Huberman, 1994).

\section{Résultats}

Six participants ont été rencontrés, soit une femme et cinq hommes dont l'âge variait de 36 à 57 ans. Trois participants ont rapporté faire partie d'une minorité visible (Noirs). En termes de trouble mental, trois participants ont déclaré avoir un trouble bipolaire, deux un épisode de dépression et un participant a refusé de répondre. Une entrevue a été réalisée en anglais. 
TABLEAU 1

Description des participants

\begin{tabular}{|l|l|l|l|l|l|}
\hline Pseudonyme & Âge & Genre & Groupe culturel/ ethnique & Niveau d'éducation & Trouble de santé mentale \\
\hline Bobby & 43 ans & Homme & Blanc & 2 $^{\text {secondaire }}$ \\
\hline Diana & 54 ans & Femme & Trinidad Tobago & Cégep & Trouble bipolaire \\
\hline Éric & 39 ans & Homme & Haïti & 3e secondaire \\
\hline Jacob & 57 ans & Homme & Québécois (Noir) & Primaire & Dépression \\
\hline Patrick & 40 ans & Homme & Québécois & 1 $^{\text {re }}$ secondaire \\
\hline Vincent & 36 ans & Homme & Québécois & 4 $^{\text {e }}$ secondaire \\
\hline
\end{tabular}

TABLEAU 2

Contacts policiers et judiciaires évoqués

\begin{tabular}{|c|c|c|c|c|c|}
\hline & Policiers & Équipes mixtes & Tribunaux réguliers & Tribunaux spécialisés & Incarcération et suivis post-détention \\
\hline Bobby & Oui & Oui & Oui & Non* & Oui \\
\hline Diana & Oui & Incertain & Oui & Non & Oui \\
\hline Éric & Oui & Non & Oui & Oui & Non \\
\hline Jacob & Oui & Oui & Oui & Oui & Oui \\
\hline Patrick & Oui & Incertain & Oui & Oui & Oui \\
\hline Vincent & Oui & Oui & Oui & Oui & Oui \\
\hline
\end{tabular}

* Le participant est sur une liste d'attente pour la participation à un tel programme. 
Le Tableau 2 synthétise les différents types de contacts policiers et judiciaires mentionnés par les participants.

L'analyse des entretiens révèle cinq thèmes. Les trois premiers sont transversaux et non propres à un type d'acteur ou de situation. Les deux autres ont trait à des interactions spécifiques, respectivement avec les services policiers et avec les services judiciaires réguliers et alternatifs.

\section{Thème 1: Des représentations complexes de l'implication judiciaire}

Bien qu'aucune question de l'entrevue n'ait porté sur les raisons de leur implication judiciaire, les personnes rencontrées ont spontanément évoqué celles-ci. Elles attribuent leur implication judiciaire à des facteurs individuels, interpersonnels et systémiques. Malgré le petit nombre de personnes rencontrées, deux profils semblent émerger: un premier où les comportements individuels sont à l'avant-plan, et un deuxième où ce sont les facteurs systémiques qui dominent.

\section{1 «À chaque jour j'étais à la bataille pour me défendre »}

Patrick ${ }^{3}$, Jacob et Bobby reconnaissent leur tendance à recourir à des comportements agressifs dans des situations stressantes ou conflictuelles: «des fois quelqu'un qui me traitait de cave je lui crissais ça entre les dents» (Bobby). Pour Patrick, cette propension s'aggrave lorsqu'il est intoxiqué, mais encore davantage lorsqu'il souffre de la faim et de la fatigue: «C'est plus en état de fatigue et de surconsommation et ne pas avoir mangé pendant une couple de jours. Un moment donné, je viens comme un animal, je viens agressif.» Les relations sociales développées dans des milieux de rue sont aussi nommées comme contribuant aux comportements criminels, car «en plus tu rencontres du monde assez spécial, du monde qui consomme, tsé?». En contrepartie, les participants indiquent que leurs relations sociales significatives avec un enfant, une conjointe ou des amis les incitent à éviter des milieux ou des comportements susceptibles d'être judiciarisés.

Si ces participants s'attribuent la responsabilité de leurs comportements, ils identifient également des évènements de leur jeunesse qui y sont associés. Patrick évoque son passage en famille d'accueil où il était mal nourri et volait de la nourriture pour lui-même et les autres enfants hébergés :

3. Tous les noms utilisés sont des pseudonymes choisis par les participants. 
Quand je voyais qu'il y avait de la bonne bouffe, y'avait des enfants aussi, y'avait des enfants à eux autres, c'était une famille d'accueil. Je volais des osti de gros gâteaux dans le congèle. [...] Pi je donnais ça à du monde qui était comme moi, tsé qui osait pas. Ils savaient là, à un moment donné que c'était moi. Fack, euh, c'est ça, je pense que c'est de là que ça à parti, comme un mode de survie, astheure ça a comme resté, tsé.

Jacob, lui, décrit une enfance et une adolescence de «bagarreur»:

J'ai perdu mes parents quand j'étais jeune, je les ai perdus à 2 ans, à 3 ans. Qu'est-ce qui va faire, un autre problème, j'avais des lunettes épaisses comme des fonds de bouteilles. Ah c'est pas drôle, j'ai passé toute ma jeunesse, je suis parti de mon école parce que à chaque jour j'étais à la bataille pour me défendre, défendre mes yeux, défendre ma vue, pi fonds de bouteille, monsieur 4 yeux pi ça a été comme ça. Pi là je suis allé en centre d'accueil jusqu'à mes 18 ans.

\section{2 «It needs better justice»}

Éric, Diana et Vincent comprennent leur implication judiciaire différemment. Leur description des raisons qui les ont amenés à être en contact avec les policiers et le système judiciaire est souvent teintée d'ambiguiité et de confusion. Parfois, les participants ne gardent aucun souvenir des gestes qui leur sont reprochés, entraînant des sentiments de doute et de confusion. Éric relate une telle situation:

On m’a dit que j'avais liché [quelqu'un]. J'ai essayé vraiment de m'en souvenir, mais je ne m'en souviens vraiment vraiment pas. On me l'a décrit parce que c'est écrit dans les rapports de police. Je ne me souviens pas de tout ça. J'ai essayé, j'ai essayé de me souvenir. À un moment donné, pourtant je ne suis pas fou, comment ça se fait. Il y a une psychologue, elle m'a expliqué, parce que j'étais pas dans mon état habituel, il faut que je l'accepte. [...] J'ai de la difficulté à me pardonner ça.

À d'autres occasions, leur version des faits diffère de celle retenue par les policiers et les autres acteurs judiciaires, entraînant des sentiments d'incompréhension et d'injustice. Diana, par exemple, explique avoir subi un procès alors qu'elle avait elle-même porté plainte à la suite d'un épisode de victimisation. Elle raconte avoir été faussement accusée d'introduction par effraction dans son propre appartement après avoir porté plainte à la police parce qu'un homme avait pris possession de son appartement. L'erreur judiciaire n'aurait été reconnue qu'après plusieurs mois dans le système carcéral. Elle attribue ce manque de crédibilité à une dynamique raciale. 
I think, [the country] needs better justice, especially for the Black people. [...] The justice should listen to the Black people in this country because a lot of us are in jail for [no] reason. If they could just take the time to hear us out and judge us right.

\section{Thème 2: "Je leur disais, pourtant»-des interactions dépourvues de justice procédurale}

Les propos rapportés dans le thème précédent évoquent un sentiment d'injustice qui transparaît dans tous les entretiens. Des situations perçues comme profondément injustes sont décrites à différents moments de leurs parcours, et au sein de différents services, systèmes et institutions. Ce sentiment d'injustice est lié aux attitudes négatives ou discriminatoires des intervenants, qu'ils soient procureurs, policiers, personnel des urgences hospitalières, agents de sécurité, médecins ou travailleurs sociaux. Plusieurs des personnes rencontrées s'expliquent les différentes situations d'injustice et de discrimination vécues par leur propre manque de crédibilité et de légitimité, souvent en lien avec les étiquettes d'itinérants, de personnes ayant des troubles mentaux ou d'utilisateurs de drogues, en plus, parfois, de leur statut de minorité visible. Elles rapportent être peu souvent entendues et encore moins souvent crues par les différents acteurs qu'elles rencontrent. Vincent, par exemple, mentionne ne pas avoir été écouté dans les services d'urgence, ce qui a amené un délai de traitement pour un problème de santé majeur:

Je leur disais, pourtant, [...] je ne suis plus capable de dormir, je shake tout le temps, le cœur m'arrête, le cœur me repart, j'ai dit je ne suis plus capable, je suis en pleine santé et je ne viendrais pas à l'hôpital pour des niaiseries, il faut que vous fassiez quelque chose et ils n'étaient pas capables.

De la même façon, Jacob relate avoir été déclaré coupable de tentative de vol, alors qu'il avait plutôt décidé de ne pas terminer sa transaction à une pharmacie après des commentaires désobligeants du commis. La juge responsable de son dossier ne l'a pas cru.

J'ai dit: «Madame la juge, tentative de vol? Je suis sorti jusqu'à les portes, euh, ça n'a pas sonné, j'avais rien sur moi, j'ai essayé d'acheter, j'ai pas aimé ça, je suis parti» et elle m’a traité de tentative de vol. J'ai passé un vraiment mauvais moment avec elle. [...] J'étais fâché, écoute, j'ai dit tous les mots, là.

Ainsi, les participants tentent fréquemment d'obtenir des services pour répondre à leurs besoins en raison de leur condition sociale, de 
même que de leurs problèmes de santé mentale et physique. Toutefois, ce recours aux services est souvent contraint par des difficultés à être pris au sérieux, à être écoutés et entendus, et donc à obtenir une réponse appropriée à leurs besoins.

Thème 3: L'accompagnement - professionnel ou non - pour prévenir et sortir de la judiciarisation

En contrepartie au thème précédent, c'est par un accompagnement «humain», «à l'écoute» et souvent «au long cours» que les personnes rencontrées entrevoient des portes de sortie à leur implication judiciaire. Des exemples de ces formes d'accompagnement vécues de façon positive sont rapportés pour chacun des stades du processus judiciaire: en amont, lors de situations de crise, lors du passage devant les tribunaux, en suivi post-détention.

\section{1 «Ça fait du bien»}

Le soutien par les intervenants de différentes équipes et divers organismes de proximité est perçu comme augmentant le bien-être et brisant l'isolement. Les liens sont très forts, voire même «comme [leur] famille» ou un "partenaire», ce qui permet aux participants de s'autoriser une certaine vulnérabilité émotive et d'être écoutés: «Des fois je pleurais avec elle, pas que j'avais pas peur de pleurer, mais on s'était tellement bien mis, comme partenaires... [...] On parlait de mes affaires et ça m'a fait du bien.»

Les suivis les plus appréciés sont ceux qui favorisent la continuité, la flexibilité et qui sont offerts de façon inconditionnelle. Un participant raconte par exemple être particulièrement reconnaissant à son intervenante d'avoir demandé une mesure exceptionnelle pour prolonger son mandat lorsque sa mère est décédée subitement, ce qui l'a aidé à traverser le deuil.

\section{2 «Ça enlève l'angoisse d'être tout seul»}

Cet accompagnement individualisé se vit aussi au tribunal. Il est particulièrement apprécié des participants. Ils se trouvent «épaulés sur comment ça marchait, [...] ce que ça veut dire pis toute». L'accompagnement à la cour permet d'obtenir des explications sur les différents processus du tribunal et d'avoir quelqu'un avec qui parler, ce qui «enlève l'angoisse 
d'être pogné tout seul. [...] [Q]uand tu vas là, t'es tout seul, c'est comme «ouf», pis là t'es perdu pis tu sais pas quoi faire.»

Si l'accompagnement est apprécié, il est parfois insuffisant. Vincent, par exemple, dit comprendre que les intervenants ne peuvent pas «faire grand-chose de plus», mais souhaiterait avoir quelqu'un «tout le temps» avec lui : «Si j'avais une blonde, si j'avais un ami tout le temps avec moi, on serait deux, on serait corrects. Mais vu que je suis seul, ben c'est ça». L'accompagnement professionnel ne peut donc compenser la profonde solitude vécue au quotidien par certains participants.

\section{Thème 4: Des perceptions mitigées du corps policier}

Si les trois thèmes précédents abordaient des facettes du vécu des participants transversales aux différents systèmes rencontrés, leurs expériences avec les policiers prennent beaucoup de place dans les récits. Les perspectives des participants quant aux policiers et à leurs interventions sont mitigées. Tous sauf un pouvaient identifier des situations où les policiers leur sont venus en aide, et mentionnent avoir vu le «côté humain» de ceux-ci. Plusieurs indiquent par ailleurs que leurs interactions sont plus positives avec le personnel des équipes spécialisées.

\section{1 «J'aurais pu faire un acte grave»}

Deux participants ont témoigné que les gestes des policiers avaient permis d'éviter une situation de crise ou son escalade. Patrick raconte que l'intervention des policiers lui avait permis d'éviter de poser des gestes graves: «Si y'avaient pas été là pour m'arrêter, j'aurais pu faire un acte grave là tsé pour le danger le monde et pour mon danger à moi.»

Pour Jacob, cet «acte grave» correspond à une tentative de suicide par procuration. Il est particulièrement reconnaissant aux policiers d'être restés calmes et de lui avoir parlé avant de le désarmer: "Eux autres étaient en train... en train de... ils voulaient me dire... moi ça ne me dérangeait pas qu'ils me tirent, je voulais qu'ils me tirent. [...] ils m'ont pas suici... ils ne m'ont pas tiré dessus.»

\section{2 «En arrière, il y avait des humains »}

La plupart des participants évoquent des moments où les policières ont pris le temps d'assurer un suivi adéquat, ont été à leur écoute et ont 
montré un réel intérêt pour leur bien-être, y compris lors d'interactions où ils étaient suspectés d'un délit. Éric dit avoir apprécié l'aide offerte par des policiers après avoir obtenu un congé de l'observation psychiatrique sans vêtements adéquats. Il raconte qu'il devait traverser la ville et que des policiers lui ont offert de le reconduire à sa destination: "Quand j'ai sorti de l'auto, je me suis penché vers celui qui conduit, il a souri, l'autre aussi [...]. J'ai senti en arrière de ces deux policiers-là, il y avait deux humains. C'est important que je le dise.»

Les pratiques policières perçues de façon positive tendent à être reliées à la transparence et la communication. Diana souligne qu'elle était reconnaissante aux policières l'ayant arrêtée pour un bris de condition de s'être assurées qu'elle comprenne bien les raisons de son arrestation. Comme Diana, Vincent a aussi apprécié avoir un échange avec les policiers lorsqu'une plainte a été faite contre lui par un voisin.

J'ai réussi à m'exprimer, ils m'ont écouté, ils ont compris les deux versions, ils ont cherché c'était où le problème, ils m’ont vraiment écouté comme il faut, j'ai vraiment été capable de parler comme il faut cette fois-là.

\section{3 «Eux autres sont cools»}

Les participants apprécient la présence d'équipes policières mixtes, comme ÉMRII, qu'ils appellent les «bons policiers». Ils considèrent que le contact avec eux est plus facile et que «y'a pas d'affaires d'abus de pouvoir, d'arrogance, pis toute», contrairement à des expériences antérieures avec les policiers. Bobby, par exemple, dit pouvoir les aborder pour leur poser des questions: «Eux autres sont cools, là! C'est quelque chose, il y a une situation que tu veux savoir, avant que ça se passe. Sont là pour ça eux, répondre aux questions.» Jacob mentionne être plus réceptif quand c'est l'intervenante d'une équipe mixte qui l'aborde pour lui parler.

\section{4 «Sont juste là pour te baisser pis toute»}

À ces exemples d'interactions positives s'opposent des perceptions négatives d'attitudes humiliantes ou discriminatoires des policiers, ou liées à l'emploi excessif de la force et à la multiplication des constats d'infraction. Tous les participants sauf un ont témoigné s'être déjà sentis abaissés ou humiliés par un policier. Plusieurs ont décrit de façon explicite ces expériences: "Sont pas obligés de t'envoyer chier comme un poisson pourri "mon bâtard, t'es un chien sale" pis toute. Sont là 
juste pour faire chier, pis te baisser, pis toute devant tes chums là. C'est pas le fun.»

Un autre participant raconte avec beaucoup de "gêne» s'être senti troublé lorsqu'un policier lui aurait «fait des blagues à caractère sexuel»: «Il disait que si je voulais de la nourriture, il fallait que je fasse quelque chose en échange... Ben là, je ne trouvais pas que c'était une blague drôle. Non, non, non.» Ces attitudes de certains policiers, qualifiées d'«arrogantes», voire d'«agressives», les font parfois réagir, ce qui entraîne l'escalade des interactions.

\section{5 «Ils essaient de te pogner»}

Certains participants, comme Patrick, décrivent leurs perceptions d'attitudes vécues comme discriminatoires, ainsi que les conséquences de celles-ci au quotidien:

Tsé ça, ça me stresse tout le temps, tsé. Ou ils passent à côté de toi super lentement pi ils te regardent, là, comme si, tsé? [...] Pi ils vont moins être porté à te suivre si t'es bien habillé, ils vont moins te voir, tsé? C'est vraiment des fois juste une question d'apparence.

Jacob témoigne avoir modifié ses habitudes et les endroits qu'il fréquente parce qu'il avait l'impression d'être l'objet d'une attention indue de la part des policiers en tant que minorité visible et qu'il n'avait pas envie de se «mettre dans le trouble», maintenant qu'il «commence une nouvelle vie». Enfin, plusieurs participants déplorent l'émission d'un grand nombre de constats d'infraction qui, pour certains d'entre eux, se chiffrent à plusieurs milliers de dollars.

\section{Thème 5: Tribunaux, prisons et suivis post-détention - Processus réguliers et alternatifs}

\section{1 «Je comprends rien »}

En général, l'expérience du passage à la cour générait de la «nervosité» ainsi que de «l'angoisse» pour les personnes rencontrées, ce qui peut devenir «épuisant [...] physiquement et mentalement». Cette angoisse est liée notamment à la difficulté qu'ont plusieurs à comprendre ce qui se passe à la cour. Bien que les avocats de l'aide juridique soient appréciés par plusieurs pour leur efficacité à minimiser les peines, certains, comme Éric, témoignent d'un besoin de mieux comprendre leur dossier: 
Y'en a [des avocats à l'aide juridique] qui sont vites. Tsé, moi j'suis slow. [...] Trop vite, moi, ça fait bobo à mes yeux. Quand c'est trop vite, euh, je ne suis pas. Je comprends rien quand c'est trop vite.

De façon similaire, Vincent trouve qu'il est «mal informé» par ses avocats et aimerait que ceux-ci prennent davantage le temps d'expliquer les différentes étapes du procès et de l'application de la sentence.

Ils devraient t'expliquer comment ça va marcher, combien de temps tu vas faire, $[. .$.$] pi rendu à ton procès, ça va être quoi ta sentence. [...] On devrait$ avoir le droit à l'information, le droit d'être bien renseignés par nos avocats.

Malgré le soutien des intervenants, certains participants avaient toujours du mal, au moment de l'entrevue, à expliquer les points majeurs de leur dossier judiciaire.

\section{2 «Sometimes you need to fight»}

De la même façon, l'incarcération et la période post-détention sont souvent marquées par la peur, la confusion et l'incertitude chez les participants. Pour Vincent, «quand je suis sorti, je ne voyais pas vraiment de possibilité de réinsertion sociale parce que je ne voyais pas de programmes, je ne voyais pas grand-chose». Diana évoque le manque de soutien offert lors d'une sortie de détention pendant un jour férié, où il n'était pas possible pour elle d'avoir accès à l'argent conservé dans son compte bancaire de la prison: «I said "I am not leaving here! Just put me back, I cannot go on the street and it's cold and I cannot go to my son right now. You guys are going to release me without nothing?"» Ayant obtenu un séjour de quelques nuits en maison de transition, elle ajoute: «Sometimes you need to fight!»

\section{3 «Le juge m'a donné un diplôme parce que j'ai réussi »}

À ces expériences difficiles des processus judiciaires «réguliers» s'opposent des perceptions plus positives des processus alternatifs. Jacob décrit «le meilleur moment que j'ai passé [à la cour] c'était le dernier. Quand le juge m’a donné un diplôme parce que j’ai réussi... [...] Ça c'est un moment significatif que j'ai retenu.»

Patrick témoigne de façon plus précise des conséquences positives de ce processus judiciaire alternatif sur différentes sphères de sa vie:

J'avais deux mille piastres de tickets. Là ils [organisme de défense des droits] m'ont aidé, pi j’avais comme changé de vie, j'avais essayé de retour- 
ner à l'école pi tout ça, pi je m'étais tout fait enlever ça, ça c'était pas mal significatif. [...] C'était un gros montant que je pouvais pas payer fack au moins ça m’a aidé, ça m’a libéré, psychologiquement.

Les participants ont donc abordé les effets positifs du passage par les processus judiciaires alternatifs sous l'angle de leurs impacts psychologiques et de la meilleure qualité de la relation avec les acteurs judiciaires. Aucun n'a abordé l'impact de ces processus alternatifs sur leurs trajectoires dans les systèmes (meilleur arrimage avec les services de santé ou incarcération évitée, par exemple).

\section{Discussion}

Le cadre théorique de la justice procédurale s'est révélé fructueux dans l'interprétation des données: d'abord, le sentiment de justice procédurale ressort non seulement dans les interactions avec des figures d'autorité formelles et déjà largement étudiées, comme les policiers et les juges, mais également dans les interactions avec de nombreuses figures d'autorité informelles (médecins, intervenants, avocats) vues par les participants. Pour les personnes rencontrées, les principes clés de la justice procédurale (écoute, respect, transparence, participation active) ont une grande importance, que ce soit dans les sphères judiciaires ou sociosanitaires. Si des processus alternatifs ou adaptés semblent améliorer le sentiment de justice procédurale, rejoignant ainsi les conclusions d'écrits antérieurs (Kopelovich, Yanos, Pratt et Koerner, 2013; Lamanna et al., 2018; Munetz, Ritter, Teller et Bonfine, 2014), ces interactions revêtent un caractère exceptionnel dans les récits des participants que nous avons rencontrés. Surtout, il semble que les interactions vécues comme justes par les participants sont celles où la personne en situation d'itinérance est identifiée par les figures d'autorité comme «vulnérable» et non comme «déviante», par exemple à sa sortie d'une hospitalisation en psychiatrie, ou du fait de sa participation à un «tribunal de santé mentale». Ici, au-delà du cadre théorique de la justice procédurale, les théories de l'étiquetage (Becker, 1963) et de l'intersectionnalité (Hill Collins et Bilge, 2016) peuvent contribuer à l'interprétation des résultats. Selon la première, on pourrait parler d'une justice procédurale conditionnelle à l'apposition d'une étiquette où les statuts d'«itinérant» ou de «délinquant» sont relégués à l'arrière-plan, et où le statut lié à la maladie ou à la vulnérabilité domine (Becker, 1963). Ce cadre théorique peut également être mobilisé pour interpréter la 
compréhension qu'ont les participants de leur propre implication judiciaire. En effet, selon Becker (1963), les personnes étiquetées en viennent parfois à intérioriser les étiquettes et à les intégrer à leur identité. On remarque que les trois participants s'attribuant la responsabilité de leur implication judiciaire ont une longue expérience d'interactions avec des institutions, entre autres en centres jeunesse ou en détention, où une étiquette de «bagarreur» leur a été accolée. Le cadre théorique de l'intersectionnalité peut, quant à lui, être utile pour comprendre comment différents systèmes d'oppression, en particulier, dans le cas présent, le racisme et le classisme, peuvent s'entrecroiser et façonner les expériences et parcours des personnes. Bien qu'un échantillon plus large et plus diversifié eût été nécessaire pour une analyse intersectionnelle approfondie, les résultats suggèrent que les dynamiques raciales et de classe sont associées de façon complexe au sentiment de justice procédurale. Dans ce contexte, il est pertinent de s'interroger sur la capacité des diverses institutions sociales à offrir des services vécus comme justes par les personnes situées au confluent d'identités sociales marginalisées, à différents moments de leurs parcours.

Les résultats permettent également de saisir comment certaines caractéristiques des interactions judiciaires, en particulier lors des comparutions, contribuent au sentiment d'injustice et d'impuissance des personnes judiciarisées. Non seulement les étiquettes qui leur sont apposées leur enlèvent la crédibilité nécessaire pour s'exprimer et être entendus sur un pied d'égalité, mais des facteurs comme la fatigue, la difficulté à bien comprendre les procédures ou à exprimer ses émotions d'une façon jugée acceptable, peuvent ajouter au manque de crédibilité. Les processus judiciaires eux-mêmes, comme l'utilisation d'un langage spécialisé et le rythme des audiences, contribuent également à l'impuissance ressentie par plusieurs participants (Sylvestre, Blomley et Bellot, 2020), diminuant d'autant plus le sentiment de justice procédurale (Livingston et al., 2016). Cela pose la question d'un accompagnement qui vient pallier ce «déficit de crédibilité» par la revendication et la défense des droits, illustrant l'importance de l'accompagnement social réalisé par différents organismes communautaires et équipes de proximité pour les personnes en situation d'itinérance ayant un trouble mental, y compris dans la sphère judiciaire.

Certaines limites doivent être considérées dans l'interprétation des résultats. D’abord, des enjeux de faisabilité (délais occasionnés par des changements de personnel au sein de l'équipe ainsi que lors de l'éva- 
luation éthique, temps limité alloué au projet par la subvention) liés au recrutement ont diminué le nombre de personnes interrogées, limitant la saturation empirique des données. Malgré le désir de l'équipe de recruter un nombre égal de femmes et d'hommes, des personnes plus jeunes ou plus âgées ainsi que des personnes autochtones ou immigrantes pour témoigner de leur expérience spécifique, les mêmes enjeux de faisabilité n'ont pas permis de diversifier l'échantillon. Des recherches futures devraient documenter comment les programmes offrant des solutions de rechange à l'arrestation ou à l'incarcération fonctionnent selon, par exemple, qu'il s'agit de femmes victimes de violence, de jeunes ayant été placés ou de personnes autochtones. Finalement, la conduite d'entretiens auprès de ce groupe pose des enjeux éthiques importants, tout particulièrement lorsque les questions abordées réfèrent à certaines expériences passées douloureuses ou sensibles (Gagnon, Beaudry et Deschenaux, 2019). Certains participants ont par ailleurs refusé de parler de leur perception de certaines expériences, notamment liées à des interactions policières. À l'inverse, il était également apparent que certains participants étaient souvent soulagés de disposer d'un espace de parole et manifestaient parfois une volonté de tisser un lien avec la personne menant les entretiens. Il est donc possible qu'un biais de désirabilité sociale ait pu influer sur la parole des personnes interrogées, notamment en adoptant les discours tenus par les intervenants sur leur parcours. Des travaux futurs pourraient, en plus d'élargir et de diversifier l'échantillon, trianguler les sources de données par l'observation des pratiques sur le terrain.

Malgré ces limites, la présente étude met de l'avant la parole de personnes qui ne sont pas nécessairement faciles à rejoindre du fait de multiples identités susceptibles d'être stigmatisées. Dans l'ensemble, les résultats illustrent à la fois les forces (renforcement du sentiment de justice procédurale, accompagnement individualisé) des processus ou programmes spécialisés et les limites de ceux-ci, qui, malgré tout, ne remettent pas en question les injustices que vivent ces personnes au quotidien.

\section{Références}

Batastini, A. B., Bolanos, A. D. et Morgan, R. D. (2014). Attitudes toward hiring applicants with mental illness and criminal justice involvement: The impact of education and experience. International Journal of Law and Psychiatry, 37(5), 524-533. doi : https://doi.org/10.1016/j.ijlp.2014.02.025 
Becker, H. S. (1963). Outsiders: Studies in the sociology of deviance. New York, NY: The Free Press.

Blais, M. et Martineau, S. (2006). L'analyse générale inductive: description d'une démarche visant à donner un sens à des données brutes. Recherches Qualitatives, 26(2), 1-18.

Caton, C. L. M., Dominguez, B., Schanzer, B., Hasin, D. S., Shrout, P. E., Felix, A.,... Hsu, E. (2005). Risk factors for long-term homelessness: Findings from a longitudinal study of first-time homeless single adults. American Journal of Public Health, 95(10), 1753-1759. doi: 10.2105/ajph.2005.063321

Charette, Y., Crocker, A. G. et Billette, I. (2011). The judicious judicial dispositions juggle: characteristics of police interventions involving people with a mental illness. Canadian Journal of Psychiatry, 56(11), 677-685.

Chesnay, C. T., Bellot, C. et Sylvestre, M.-E. (2013). Taming disorderly people one ticket at a time: The penalization of homelessness in Ontario and British Columbia. Canadian Journal of Criminology and Criminal Justice, 55(2), 161-185.

Choe, J. Y., Teplin, L. A. et Abram, K. M. (2008). Perpetration of violence, violent victimization, and severe mental illness: Balancing public health concerns. Psychiatric Services, 59(2), 153-164. doi : 10.1176/appi.ps.59.2.153

Commission des droits de la personne et des droits de la jeunesse du Québec. (2009). La judiciarisation des personnes itinérantes à Montréal: un profilage social. Repéré à http://www.cdpdj.qc.ca/publications/itinerance_avis.pdf

Copeland, L. A., Miller, A. L., Welsh, D. E., McCarthy, J. F., Zeber, J. E. et Kilbourne, A. M. (2009). Clinical and demographic factors associated with homelessness and incarceration among VA patients with bipolar disorder. American Journal of Public Health, 99(5), 871-877.

Coroner du Québec. (2012). Rapport d'enquête du Dr Jean Brochu, coroner, sur les décès de MM. Patrick Limoges et Mario Hamel. Montréal, Québec: Gouvernement du Québec.

Coroner du Québec. (2016). Rapport d'enquête de Me Luc Malouin, coroner, sur les causes et les circonstances du décès de Alain Magloire. Montréal, Québec: Gouvernement du Québec.

Crawford, D. M., Whitbeck, L. B. et Hoyt, D. R. (2011). Propensity for violence among homeless and runaway adolescents: An event history analysis. Crime o Delinquency, 57(6), 950-968. doi: 10.1177/0011128709335100

Dollar, C. B., Ray, B., Hudson, M. K. et Hood, B. J. (2018). Examining changes in procedural justice and their influence on problem-solving court outcomes. Behavioral Sciences and the Law, 36(1), 32-45. doi : 10.1002/bsl.2329

Edalati, H., Nicholls, T. L., Schütz, C. G., Somers, J. M., Distasio, J., Aubry, T. et Crocker, A. G. (2020). Examining the relationships between cumulative childhood adversity and the risk of criminal justice involvement and victimization among homeless adults with mental illnesses after receiving housing first intervention. The Canadian Journal of Psychiatry, 65(6), 409-417. doi : 10.1177/0706743720902616

Fortin, V. et Raffestin, I. (2017). Le Programme d'accompagnement justiceitinérance à la cour municipale de Montréal (PAJIC): un tribunal spécialisé 
ancré dans le communautaire. Revue générale de droit, 47, 177-208. doi: https://doi.org/10.7202/1040522ar

Frappier, A., Vigneault, L. et Paquet, S. (2009). À la fois malade et criminalisé: témoignage d'une double marginalisation. Santé mentale au Québec, 34(2), 21-30. doi: https://doi.org/10.7202/039123ar

Frounfelker, R. L., Glover, C. M., Teachout, A., Wilkniss, S. M. et Whitley, R. (2010). Access to supported employment for consumers with criminal justice involvement. Psychiatric Rebabilitation Journal, 34(1), 49-56.

Furness, T., Maguire, T., Brown, S. et McKenna, B. (2016). Perceptions of procedural justice and coercion during community-based mental health crisis: A comparison study among stand-alone police response and coresponding police and mental health clinician response. Policing: A Journal of Policy and Practice, 11(4), 400-409. doi : 10.1093/police/paw047

Gagnon, M., Beaudry, C. et Deschenaux, F. (2019). «Prendre soin» des participants lors d'entretiens réalisés en contexte de recherches sensibles. Recherches qualitatives, 38(2), 71-92. doi : https://doi.org/10.7202/1064931ar

Guiresse, G., Senon, J.-L. et Rossini, K. (2019). Les tribunaux de santé mentale américains, un exemple de programme de déjudiciarisation. Annales Médicopsychologiques, revue psychiatrique, 177(9), 896-901. doi: https://doi.org/ 10.1016/j.amp.2019.08.008

Hartford, K., Heslop, L., Stitt, L. et Hoch, J. S. (2005). Design of an algorithm to identify persons with mental illness in a police administrative database. International Journal of Law and Psychiatry, 28(1), 1-11. doi: http://dx.doi. org/10.1016/j.ijlp.2004.12.001

Hill Collins, P. et Bilge, S. (2016). Intersectionality. Oxford, Royaume-Uni: Polity Press.

Kisely, S., Campbell, L. A., Peddle, S., Hare, S., Pyche, M., Spicer, D. et Moore, B. (2010). A controlled before evaluation of a mobile crisis partnership between mental health and police services in Nova Scotia. Canadian Journal of Psychiatry, 55(10), 662-668.

Kopelovich, S., Yanos, P., Pratt, C. et Koerner, J. (2013). Procedural justice in mental health courts: Judicial practices, participant perceptions, and outcomes related to mental health recovery. International Journal of Law and Psychiatry, 36(2), 113-120. doi : https://doi.org/10.1016/j.ijlp.2013.01.004

Lamanna, D., Shapiro, G. K., Kirst, M., Matheson, F. I., Nakhost, A. et Stergiopoulos, V. (2018). Co-responding police-mental health programmes: Service user experiences and outcomes in a large urban centre. International Journal of Mental Health Nursing, 27(2), 891-900. doi : 10.1111/inm.12384

Leclair, M. C., Lemieux, A. J., Roy, L., Martin, M. S., Latimer, E. A. et Crocker, A. G. (2020). Pathways to recovery among homeless people with mental illness: Is impulsiveness getting in the way? The Canadian Journal of Psychiatry, 65(7), 473-483. doi : 10.1177/0706743719885477

Levitt, A. J., Culhane, D. P., DeGenova, J., O'Quinn, P. et Bainbridge, J. (2009). Health and social characteristics of homeless adults in Manhattan who were chronically or not chronically unsheltered. Psychiatric Services, 60(7), 978-981. 
Livingston, J. D. (2016). Contact between police and people with mental disorders: A review of rates. Psychiatric Services, 67(8), 850-857. doi: 10.1176/appi.ps.201500312

Livingston, J. D., Crocker, A. G., Nicholls, T. L. et Seto, M. C. (2016). Forensic mental health tribunals: A qualitative study of participants' experiences and views. Psychology, Public Policy, and Law, 22(2), 173-184. doi: 10.1037/ law0000084

Lurigio, A. J. (2012). Responding to the needs of people with mental illness in the criminal justice system: An area ripe for research and community partnerships. Journal of Crime and Justice, 35(1), 1-12. doi : 10.1080/ 0735648X.2011.637402

Lynch, S. M., DeHart, D. D., Belknap, J. E., Green, B. L., Dass-Brailsford, P., Johnson, K. A. et Whalley, E. (2014). A multisite study of the prevalence of serious mental illness, PTSD, and substance use disorders of women in jail. Psychiatric Services, 65(5), 670-674. doi : 10.1176/appi.ps.201300172

Mahoney, M. K. (2014). Procedural justice and the judge-probationer relationship in a co-occurring disorders court. International Journal of Law and Psychiatry, 37(3), 260-266. doi: https://doi.org/10.1016/j.ijlp.2013.11.022

Markowitz, F. E. (2011). Mental illness, crime, and violence: Risk, context, and social control. Aggression and Violent Behavior, 16(1), 36-44. doi: http:// dx.doi.org/10.1016/j.avb.2010.10.003

Maxwell, J. A. (2005). Qualitative research design: An interactive approach. Thousand Oaks, CA: Sage Publications.

Miles, M. B. et Huberman, A. M. (1994). Qualitative data analysis: An expanded sourcebook ( $2^{\mathrm{e}}$ éd.). Thousand Oaks, CA: Sage Publications.

Munetz, M. R., Ritter, C., Teller, J. L. S. et Bonfine, N. (2014). Mental health court and assisted outpatient treatment: Perceived coercion, procedural justice, and program impact. Psychiatric Services, 65(3), 352-358. doi: 10.1176/appi.ps.002642012

Nault, G., Couture-Glassco, É. et Larose-Hébert, K. (2016). Le mal caché de la rue: le poids de l'étiquette. Reflets: revue d'intervention sociale et communautaire, 22(1), 56-82.

Neergaard, M. A., Olesen, F., Andersen, R. S. et Sondergaard, J. (2009). Qualitative description - the poor cousin of health research? BMC Medical Research Methodology, 9(1), 52. doi: 10.1186/1471-2288-9-52

Poremski, D., Woodhall-Melnik, J., Lemieux, A. et Stergiopoulos, V. (2015). Persisting barriers to employment for recently housed adults with mental illness who were homeless. Journal of Urban Health, 93(1), 96-108. doi: 10.1007/s11524-015-0012-y

Prins, S. J. (2014). Prevalence of mental illnesses in U.S. state prisons: A systematic review. Psychiatric Services, 65(7), 862-872. doi: 10.1176/appi. ps.201300166

Provost, J. (2011). Programme d'Accompagnement Justice et Santé mentale «PAJ-SM» à la cour municipale de la Ville de Montréal - Bilan et perspectives. Montréal, Québec: Cour municipale de Montréal. 
Roy, L., Crocker, A. G., Nicholls, T. L., Latimer, E. A. et Ayllon, A. R. (2014). Criminal behavior and victimization among homeless individuals with severe mental illness: A systematic review. Psychiatric Services, 65(6), 739750. doi: 10.1176/appi.ps.201200515.

Roy, L., Crocker, A. G., Nicholls, T. L., Latimer, E., Gozdzik, A., O’Campo, P. et Rae, J. (2016). Profiles of criminal justice system involvement of mentally ill homeless adults. International Journal of Law and Psychiatry, 45, 75-88. doi: http://dx.doi.org/10.1016/j.ijlp.2016.02.013

Roy, L., Lemieux, A. J. et Crocker, A. (2019). L'expérimentation sociale et la judiciarisation: constats et enjeux du projet Chez soi. Dans T. Benmarhnia, P.-M. David et B. Godrie (dir.), Les sociétés de l'expérimentation: enjeux épistémologiques, éthiques et politiques (p. 141-158). Montréal, Québec: Presses de l'Université du Québec.

Sandelowski, M. (2010). What's in a name? Qualitative description revisited. Research in Nursing and Health, 33(1), 77-84. doi : 10.1002/nur.20362

Schneider, R. D., Crocker, A. G. et Leclair, M. C. (2016). Mental health courts and diversion programs. Dans J. A. Chandler et C. M. Flood (dir.), Law and mind: Mental healtb law and policy in Canada (p. 303-323). Lexis Nexis Canada.

St-Jacques, B. (2016). Profilage social et judiciarisation des personnes itinérantes à Montréal - Opération Droits Devant et autres consolations! Reflets, 22(1), 173-182.

Sylvestre, M.-E. (2010). Disorder and public spaces in Montreal: Repression (and resistance) through law, politics, and police discretion. Urban Geography, 31(6), 803-824. doi : 10.2747/0272-3638.31.6.803

Sylvestre, M.-È., Bellot, C. et Chesnay, C. (2012). De la justice de l'ordre à la justice de la solidarité: une analyse des discours légitimateurs de la judiciarisation de l'itinérance au Canada. Droit et société, 81(2), 299-320.

Sylvestre, M.-E., Bellot, C., Ménard, P. A. C. et Tremblay, A. C. (2011). Le droit est aussi une question de visibilité : l'occupation des espaces publics et les parcours judiciaires des personnes itinérantes à Montréal et à Ottawa. Canadian Journal of Law E Society/La Revue Canadienne Droit et Société, 26(3), 531-561.

Sylvestre, M.-E., Bernier, D. et Bellot, C. (2015). Zone restriction orders in Canadian courts and the reproduction of socio-economic inequality. Onati Socio-legal Series, 5(1), 280-297.

Sylvestre, M.-E., Blomley, N. et Bellot, C. (2020). Red zones: Criminal law and the territorial governance of marginalized people. Cambridge, Royaume-Uni: Cambridge University Press.

Tyler, T. R. (1990). Why people obey the law. New Haven, CT: Yale University Press.

Tyler, T. R. (2009). Legitimacy and criminal justice: The benefits of selfregulation. Obio State Journal of Criminal Law, 7, 307-359.

Tyler, T. R. et Fagan, J. (2008). Legitimacy and cooperation: Why do people help the police fight crime in their communities. Obio State Journal of Criminal Law, 6, 231-275. 
Wales, H. W., Hiday, V. A. et Ray, B. (2010). Procedural justice and the mental health court judge's role in reducing recidivism. International Journal of Law and Psychiatry, 33(4), 265-271. doi: https://doi.org/10.1016/j. ijlp.2010.06.009

Welsh, M. et Abdel-Samad, M. (2018). You're an embarrassment: Un-housed people's understandings of policing in downtown San Diego. Criminology, Criminal Justice, Law er Society, 19, 33-49.

Wood, J. D. et Watson, A. C. (2016). Improving police interventions during mental health-related encounters: past, present and future. Policing and Society, 27(3), 289-299. doi : 10.1080/10439463.2016.121

\title{
Homelessness, mental health and justice: Experiences and perceptions of service users in Montréal
}

\begin{abstract}
Several programs and agencies have been developed and implemented in attempts to adapt police and legal processes to the needs of people living with mental illness, especially those who experience homelessness. The present qualitative descriptive study examines how homeless people with mental illness experience police and justice-related services. Analysis of six interviews reveals complex attitudes toward involvement with these services, including feelings of responsibility and injustice; the perception that most programs and agencies are not fulfilling their mandates, although this failure is sometimes counterbalanced by positive experiences with community-based support programs; and different views of those services considered to be either "regular" or "alternative" processes. Participants emphasized the principles of procedural justice - in particular what procedures labelled "alternative" offered in this regard, although they noted that even these interactions were unlikely to fully respect these principles. Our results raise questions about the ability of different social institutions to offer services that will be considered fair by individuals who experience convergent marginalized identities and are at different stages along their personal trajectories.
\end{abstract}

KEYWORDS - Homelessness, criminal justice involvement, police, mental illness.

\section{Indigencia, salud mental, justicia. Experiencia y percepciones de los usuarios de servicios en Montreal}

RESUMEN - Varios programas y servicios han sido desarrollados con el fin de adaptar las intervenciones policiales y los procesos judiciales a las necesidades de las personas con problemas de salud mental, quienes además están en situación de calle. Este estudio adopta una aproximación cualitativa descriptiva con el fin de explorar la experiencia de estos servicios de las personas que viven a la vez una situación de calle, y un problema de salud mental. El análisis de seis entrevistas revela las representaciones complejas, entre sentimiento de responsabilidad e injusticia, de los participantes acerca de su implicación judicial; la falta de legitimidad vivida en la mayor parte de sus interacciones, a la cual el acompañamiento le ofrece a veces un contra peso; $y$, final- 
mente, las percepciones diferenciales de los servicios según su naturaleza "regular" o "alternativa". Los participantes ponen en el primer plano de sus narrativas los principios de la justicia procedural, en particular lo que permiten a este respecto los llamados procesos "alternativos", pero, igualmente, el carácter excepcional de las interacciones que respetan estos principios. Los resultados nos llevan a cuestionar la capacidad por parte de diversas instituciones sociales de ofrecer servicios vividos como justos por las personas situadas en la confluencia de identidades sociales marginalizadas, en diferentes momentos de sus trayectorias.

PALABRAS CLAVE - Indigencia, judicialización, policía, problemas mentales. 